\title{
Assessments of Arterial Stiffness and Endothelial Function Using Pulse Wave Analysis
}

\author{
Lee Stoner, ${ }^{1}$ Joanna M. Young, $^{2,3}$ and Simon Fryer ${ }^{4}$ \\ ${ }^{1}$ School of Sport and Exercise, Massey University, P.O. Box 756, Wellington 6140, New Zealand \\ ${ }^{2}$ Lipid and Diabetes Research Group, Diabetes Research Institute, Christchurch Hospital, Christchurch 8011, New Zealand \\ ${ }^{3}$ Department of Medicine, University of Otago, Christchurch 8140, New Zealand \\ ${ }^{4}$ School of Sciences and Physical Education, University of Canterbury, Christchurch 8140, New Zealand \\ Correspondence should be addressed to Lee Stoner, dr.l.stoner@gmail.com
}

Received 29 September 2011; Revised 16 February 2012; Accepted 2 March 2012

Academic Editor: Robert M. Schainfeld

Copyright ( $) 2012$ Lee Stoner et al. This is an open access article distributed under the Creative Commons Attribution License, which permits unrestricted use, distribution, and reproduction in any medium, provided the original work is properly cited.

Conventionally, the assessments of endothelial function and arterial stiffness require different sets of equipment, making the inclusion of both tests impractical for clinical and epidemiological studies. Pulse wave analysis (PWA) provides useful information regarding the mechanical properties of the arterial tree and can also be used to assess endothelial function. PWA is a simple, valid, reliable, and inexpensive technique, offering great clinical and epidemiological potential. The current paper will outline how to measure arterial stiffness and endothelial function using this technique and include discussion of validity and reliability.

\section{Introduction}

Cardiovascular disease (CVD), the leading cause of mortality in the Western world [1], has a very long asymptomatic phase of development, starting as early as the first decade of life [2]. It is imperative, therefore, that clinical scientists and epidemiologists have at their disposal simple, valid, and reliable techniques to assess and track the progression of CVD. Noninvasive assessment techniques fall under two broad categories: those that assess endothelial health and those that assess arterial stiffness. Assessment of endothelial function indicates the functional health of the vascular system, whereas arterial stiffness assesses structural characteristics. Together, these techniques may provide complimentary indices of CVD risk.

Conventionally, assessments of endothelial function and arterial stiffness require different sets of equipment, making the inclusion of both tests impractical for clinical and epidemiological studies. Pulse wave analysis (PWA) is a simple and noninvasive technique that has been widely used in epidemiological [3] and interventional studies [4]. PWA provides useful information regarding the mechanical properties of the arterial tree and the ventricular-vascular interaction [5] and can also be used to assess endothelial function [6]. PWA is a simple, valid, reliable, and inexpensive technique, offering great clinical and epidemiological potential. The current review will outline how to measure arterial stiffness and endothelial function using this technique and include discussion of validity and reliability.

\section{Arterial Stiffness}

Arterial stiffness is a general term that collectively describes distensiblility, compliance, and elastic modulus of the arterial vascular system. These properties are not homogenous along the arterial tree and muscular and elastic vessels differ. Arterial stiffness can be measured systemically, regionally, or locally. Local measurements provide important physiological information and are more quantitative and sensitive than systemic indices. However, these measurements give no indication of how the artery of interest interacts with central function (i.e., the heart) as part of an integrative system. Regional arterial stiffness is measured at arterial sites of major physiologic importance such as the aorta where the arterial buffering function is principally expressed, or a particular limb. Systemic arterial stiffness affects the global buffering properties of the arterial system, just as arterial 
blood pressure can be considered as a global value of hemodynamic load, systemic arterial stiffness reflects the overall opposition of large arteries to the pulsatile effects of ventricular ejection.

A number of methodologies have been applied to the in vivo assessment of arterial stiffness. These methodologies fall into three broad groups: (1) relating change in area of an artery to distending pressure, that is, local arterial stiffness, (2) measuring pulse wave velocity, that is, regional arterial stiffness, and (3) pulse wave analysis, that is, systemic arterial stiffness. Ultrasound and magnetic resonance imaging (MRI) are capable of measuring local arterial stiffness [21-23] as well as pulse wave velocity [24-30], but these methodologies require expensive equipment (especially in the case of MRI) and a high level of technical expertise and are often impractical within the clinical or epidemiological setting. PWV can also be assessed using dedicated equipment, including oscillometric [31-34], tonometric [32, 35-37], volume plethysmographic [38, 39], and photo plethysmographic [40-43] devices. These devices either measure the pulse wave at two peripheral sites or record the electrocardiogram and measure the pulse wave at a peripheral site, to estimate the regional arterial stiffness. Alternatively, a number of devices are also available to estimate systemic arterial stiffness using PWA.

2.1. Measurement. A number of commercial devices are available to automate PWA assessments, including Compilor (tonometric device) [44, 45], Sphygmocor (tonometric device) [44, 46-49], PulsePen (tonometric device) [50, 51], ARCSolver [47, 52] (oscillometric device), Arteriograph [33, 53-57] (oscillometric device), Omron (oscillometric device) $[55,58]$, PulseCore $[59,60]$ (oscillometric device), Viscorder [32, 34, 49] (oscillometric device), and PulseTrace $[6,42,48,61]$ (photoplethysmographic device). Applanation tonometry is considered the "gold standard" and is the most widely used technique [62]. A probe is conventionally placed on the skin overlying the radial artery, and pressure is applied to distort or applanate (flatten) the artery, creating a signal which approximates arterial pressure. The peak and trough of the radial pulse wave correspond, respectively, to systolic and diastolic blood pressure measured conventionally on the brachial artery, since blood pressure is practically identical in brachial and radial arteries [63]. Mean blood pressure is determined by integration of the radial wave. A generalized transfer factor is then used to generate the corresponding central arterial waveform [64-67].

Figure 1 shows typical features of the aortic pulse pressure waveform, from which can be derived augmentation pressure (AP), augmentation index (AIx), and arrival time of reflected waves at the central aorta $(T r) . T r$ represents the time from the onset of the ejected pulse waveform to the onset of the reflected wave and reflects aortic pulse wave velocity [68]. AP is the additional aortic systolic pressure generated by the return of the reflected waves at the central aorta, expressed in absolute terms [62]. AIx is the $\mathrm{AP}$ as a percentage of central pulse pressure and is a composite measure of aortic wave reflection and systemic

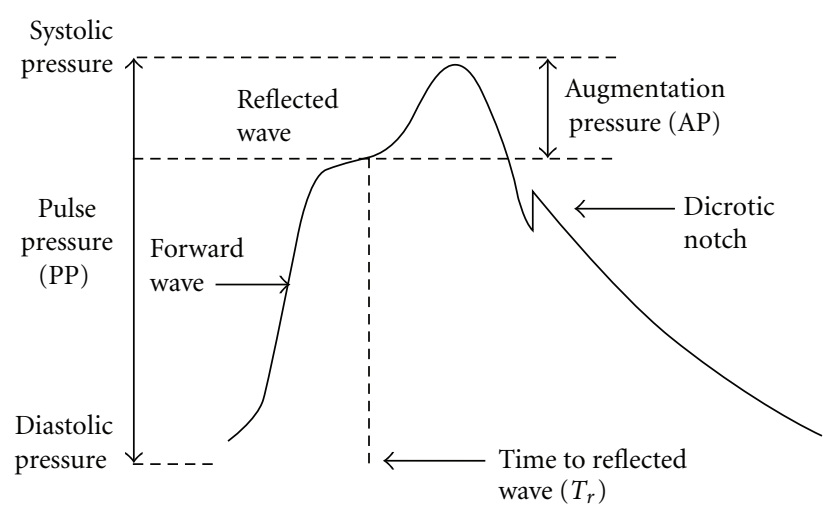

Figure 1: Aortic pulse pressure waveform. Systolic and diastolic pressures are the peak and trough of the waveform. Augmentation pressure is the additional pressure added to the forward wave by the reflected wave. Augmentation index is defined as the augmentation pressure as a percentage of pulse pressure. The dicrotic notch represents closure of the aortic valve and is used to calculate ejection duration. Time to reflection is calculated as the time at the onset of the ejected pulse waveform to the onset of the reflected wave.

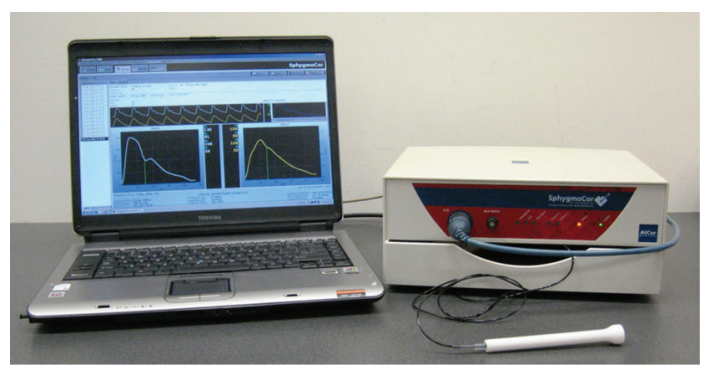

Figure 2: Electronics module (SphygmoCor device, AtCor Medical, Sydney, Australia).

arterial stiffness $[62,69]$. Although the timing of the arrival of the reflected wave at the proximal aorta is largely determined by large artery PWV, AIx is not interchangeable with PWV. It is influenced by vasoactive drugs independently of PWV [70], suggesting that it is also determined by the intensity of wave reflection, which, in turn, is determined by the diameter and elasticity of small arteries and arterioles. A number of variables are known to influence AIx. AIx increases with MAP [71] and is inversely related to body height [72] and heart rate [73, 74], with a $10 \mathrm{bpm}$ increase in heart rate resulting in a $4 \%$ reduction in AIx [73]. AIx should be normalized for a heart rate of 75 beats per minute (AIx@HR75). One of the most widely used devices, SphygmoCor, automatically adjusts the AIx at an inverse rate of $4.8 \%$ for each $10 \mathrm{bpm}$ increment. The AIx@HR75 is only calculated when the patient's heart rate is between 40 and $110 \mathrm{bpm}$. Outside of this range the software will display an $\mathrm{N} / \mathrm{C}$ indicating that no calculation was possible.

Using the SphygmoCor to illustrate the procedure (a typical setup is shown in Figure 2), PWA takes approximately 20 minutes to complete. Following at least 10 minutes supine rest, brachial artery systolic and diastolic blood pressures are measured in the nondominant arm and used to calibrate 
the PWA measurements taken on the radial artery. Radial artery waveforms are then recorded in duplicate. A highfidelity tonometer is used to obtain pressure waveforms by applying gentle pressure over the nondominant radial artery and repositioning the device until the greatest pulse signal is detected. Data is collected directly into a personal computer, and recordings are assessed visually to ensure that the best possible recording is obtained. After 20 sequential waveforms are acquired, an averaged peripheral waveform is generated and a corresponding aortic waveform is derived (see Figure 3). When consecutive AIx@HR75 readings differ by more than $4 \%$, a third reading is obtained, and the mean of the closest two readings is taken.

2.2. Validity. There is evidence that increased aortic wave reflections have adverse effects on ventricular afterload and coronary perfusion, and their pathological role has been demonstrated in several diseases [75-78]. Furthermore, increased central arterial wave reflections have been shown to independently predict cardiovascular risk and mortality $[5,79,80]$. Increased amplitude and the earlier return of the reflected wave within the cardiac cycle augments the central systolic blood pressure, resulting in increased wave reflections [69]. The amplitude and timing of reflected pressure waves are determined primarily by vascular elasticity, peripheral vascular resistance, heart rate, and left ventricle function [81].

2.3. Reliability. Any valid technique utilised for the measurement of physiological variables must be reproducible [82]. A high intra- and interobserver reproducibility of baseline AIx has been observed in healthy controls and patients with cardiovascular disease and renal dysfunction [7, 38, 82-94]. Good reproducibility of baseline time to reflection $(\mathrm{Tr})$, an alternative index to $\mathrm{AIx}$, has been reported in several studies $[88,91,94]$. However, the majority of these trials examined the reproducibility using simple BlandAltman analysis, whereas studies using more definitive intraclass correlation coefficient (ICC) and the contribution of variance components are limited $[81,82,91,93,94]$. ICC values for repeated measurements taken at hourly or weekly intervals have been reported to be $0.72-0.90$ for AIx [91, 94], 0.90 for $\mathrm{AP}[81]$, and $0.43-0.84$ for $\operatorname{Tr}[81,91,94]$. In addition, few trials have reported the reproducibility of heart rate corrected AIx $[82,91,93]$, although it is frequently used in wave reflection studies. While this technique potentially offers a valid and reliable marker of CVD risk, further study is required to determine sample size recommendations for AIx normalized to heart rate.

2.4. Recommendations. The PWA technique is particularly suitable for incorporation into clinical trials. Strengths of this technique include simplicity of assessment, relatively short training requirements for investigators, low time commitment for subjects, noninvasiveness, portability, and cost-effectiveness. Both AIx and AP confer similar reproducibility and have been reported as more reliable than $\mathrm{Tr}$. Since AIx is strongly influenced by heart rate, both AIx

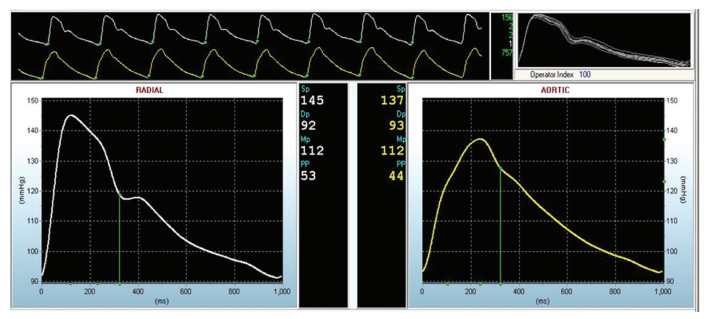

FIGURE 3: Radial artery applanation tonometry recording. The upper long panel shows the radial pressure waveform above the derived central pressure waveform. The upper right panel shows the overlaid radial waveforms, including the operator index, and the middle panel shows the quality control indices. The bottom left panel demonstrates a magnified radial arterial waveform. Systolic and diastolic pressures are 145/92 $\mathrm{mmHg}$. The bottom right panel provides a magnified derived central pressure waveform. Central pressure is $137 / 93 \mathrm{mmHg}$.

and AP should be corrected for this confounding factor [73]. Further study is warranted to determine whether the three indices of arterial stiffness provide additive prognostic value.

\section{Endothelial Health}

Functionally, the endothelium is a large autocrine, paracrine, and endocrine organ that plays a key role in vascular homeostasis [95]. Endothelial dysfunction is a pivotal, yet potentially reversible, step that has been shown to precede and predict overt CVD [96]. The endothelium has been recognized for the important role it plays in regulating vascular reactivity via the release of dilator mediators, including nitric oxide (NO) [97-100], prostaglandins [101], and endothelial-derived hyperpolarizing factor [102, 103]. The capacity of the endothelium to regulate vascular tone (reactivity) is used to confirm the health of the endothelium.

Established methodologies for evaluating peripheral endothelial function include strain-gauge venous occlusion forearm plethysmography [104, 105], ultrasound-measured flow-mediated dilation (FMD) [105-107], peripheral arterial tonometry (e.g., using the EndoPat device) [105], and laser Doppler flowmetry [105]. These techniques assess the vasodilator responses to endothelium-dependent stimuli such as acetylcholine and increased shear stress and to endothelium-independent stimuli, including sodium nitroprusside and glyceryl trinitrate (GTN). The two most commonly used techniques are strain-gauge plethysmography and FMD, with FMD being considered the "gold standard" for assessing endothelial function. FMD is a noninvasive, valid [10-13], and moderately reliable $[14,15]$ technique but is expensive and highly technical (Table 1). Strain-gauge plethysmography also offers acceptable reliability [17-20] but is an invasive technique when coupled with intraarterial infusion of vasodilators (Table 1). As such, these techniques are often impractical for use in clinical trials or 
TABLE 1: Comparison of noninvasive techniques for assessing endothelial function.

\begin{tabular}{|c|c|c|c|c|c|c|c|}
\hline Technique & Equipment & Cost & Skill level & Test time & Validity & Reliability & Ref \\
\hline PWA & $\begin{array}{l}\text { Applanation } \\
\text { tonometry }\end{array}$ & $\begin{array}{l}\text { Medium } \\
\text { US } \$ 15,000\end{array}$ & Low & 35 mins & Medium* & $\begin{array}{l}\text { Medium-high* } \\
d=0.9-2.3 \%\end{array}$ & {$[6-9]$} \\
\hline FMD & $\begin{array}{l}\text { (i) Ultrasound } \\
\text { (ii) Tourniquet }\end{array}$ & $\begin{array}{l}\text { High } \\
>\text { US } \$ 50,000\end{array}$ & High & $20 \mathrm{mins}$ & High & $\begin{array}{l}\text { Medium } \\
C V: 14-50 \%\end{array}$ & [10-16] \\
\hline Plethysmography & Strain-gauge & $\begin{array}{l}\text { Low } \\
\text { US } \$ 10,000\end{array}$ & Med. & 30 mins & Medium & $\begin{array}{l}\text { Medium } \\
C V: 8-27 \%\end{array}$ & [17-20] \\
\hline
\end{tabular}

FMD: flow-mediated dilation; CV: coefficient of variation; $d$ : sample bias (mean difference); PWA: pulse wave analysis. ${ }^{*}$ Further study is needed to corroborate these findings.

epidemiological studies. Alternatively, a limited number of studies have used PWA to assess endothelial function.

3.1. Measurement. Endothelial function can be assessed noninvasively by evaluating the effects of inhaled salbutamol on the AIx [6-8]. AIx is a measure of systemic arterial stiffness [62]. Notably, NO, considered the central molecule governing endothelial function [97-100], is a key modulator of arterial stiffness [108]. Chowienczyk et al. [6] demonstrated that salbutamol, a $\beta 2$ agonist and endotheliumdependent vasodilator, in part reduces wave reflection by activation of the L-arginine-NO pathway.

The test takes approximately 35 minutes to complete; following at least 10 minutes supine rest, baseline PWA is recorded as described above, and PWA recordings are then repeated after $5,10,15$, and 20 minutes after the administration of $400 \mu \mathrm{g}$ inhaled salbutamol. The maximal decrease in AIx from baseline following a salbutamol challenge is used as an index of endothelial function. Endotheliumindependent function can also be assessed using PWA by measurement of the reduction in AIx following sublingual GTN administration.

3.2. Validity. Wilkinson et al. [7] and Hayward et al. [8] evaluated the effects of inhaled salbutamol and sublingual GTN on the AIx. Investigators observed a significant correlation between the salbutamol-mediated reductions in AIx and increase in forearm blood flow during infusion of acetylcholine that was abolished by coadministration of NGmonomethyl-L-arginine (L-NMMA), an endothelial NO synthase inhibitor, suggesting that this may represent a valid approach for assessing endothelial function. Conversely, coadministration of intravenous L-NMMA has no influence on sublingual GTN-mediated reductions in AIx, consistent with an endothelium-independent effect [37, 74]. To date, relatively few clinical studies have employed PWA to assess endothelial function. However, preliminary validation studies have demonstrated blunted AIx responses to salbutamol in subjects exhibiting diabetes [6], hypercholesterolaemia [7], coronary artery disease [8], and peripheral vascular disease [9] among others.

3.3. Reliability. Studies examining the reproducibility of salbutamol-mediated effects on AIx are limited [7, 8, 82].
Hayward et al. [8] assessed reliability in healthy subjects using Bland-Altman analysis and reported excellent between-day mean difference $(d=0.9 \pm 2 \%)$. Wilkinson et al. [7] also assessed reliability in healthy subjects using BlandAltman analysis and reported similar findings; betweenday mean difference in the AIx response was $-2.3 \pm$ 3.0 for salbutamol-mediated changes and $0.2 \pm 6.5$ for glyceryl trinitrate (GTN)-mediated changes. More recently, Paul et al. [82] produced discrepant findings when they assessed within-day reliability using the more definitive ICC method [109]. Relatively low reliability was reported for control (ICC: 0.18) and chronic heart failure groups (CHF) (ICC: 0.04). However, relatively low reliability was also reported for GTN-mediated changes in AIx for both control (ICC: 0.58 ) and CHF groups (ICC: 0.17 ). Endothelial function assessments were repeated at hourly intervals, and the authors suggest that the low ICC values may reflect diurnal variability or a carryover effect from the first assessment. Further study is warranted to determine the reliability and sample size requirements for this promising technique.

3.4. Recommendations. Salbutamol-mediated effects on AIx can be used to estimate endothelial function. This technique offers a number of advantages, namely simplicity of assessment, relatively short training requirements for investigators, low time requirement, portability, cost-effectiveness, ease of use, good reliability indicated by preliminary studies, and the salbutamol challenge isolating the NO pathway. However, more clinical studies are required to further validate this test. Also, while NO is unarguably an important molecule governing endothelial function, the endothelium can release additional complimentary or compensatory molecules [110], most notably prostacyclin [101] and endothelial-derived hyperpolarizing factor $[102,103]$. The relative importance of these molecules varies by vascular bed and between individuals, particularly between individuals exhibiting a number of diseased states [111-120]. Therefore, while this test can provide a snapshot of endothelial function it may not provide a complete picture. Further study is warranted to determine whether baseline AIx (systemic arterial stiffness) and AIx following salbutamol inhalation confer additive prognostic value. Further study is also required to clarify sample size recommendations for this test. 


\section{Conclusion}

PWA is a simple technique capable of assessing systemic arterial stiffness and endothelial function. This test is particularly suitable for clinical and epidemiological studies. Further study is required to determine: (1) sample size requirements, and (2) whether baseline AIx (systemic arterial stiffness) and AIx following salbutamol inhalation confer additive prognostic value.

\section{References}

[1] American Heart Association, eart Disease and Stroke Statistics-2006 Update, American Heart Association, 2006.

[2] G. S. Berenson, S. R. Srinivasan, W. Bao, W. P. Newman III, R. E. Tracyand, and W. A. Wattigney, "Association between multiple cardiovascular risk factors and atherosclerosis in children and young adults. The Bogalusa Heart Study," The New England Journal of Medicine, vol. 338, no. 3, pp. 16501656, 1998.

[3] C. Vlachopoulos, K. Aznaouridis, M. F. O’Rourke, M. E. Safar, K. Baou, and C. Stefanadis, "Prediction of cardiovascular events and all-cause mortality with central haemodynamics: a systematic review and meta-analysis," European Heart Journal, vol. 31, no. 15, pp. 1865-1871, 2010.

[4] B. Williams, P. S. Lacy, S. M. Thom et al., "Differential impact of blood pressure-lowering drugs on central aortic pressure and clinical outcomes: principal results of the Conduit Artery Function Evaluation (CAFE) study," Circulation, vol. 113, no. 9, pp. 1213-1225, 2006.

[5] C. Vlachopoulosand and M. O'Rourke, "Genesis of the normal and abnormal arterial pulse," Current Problems in Cardiology, vol. 25, no. 5, pp. 303-367, 2000.

[6] P. J. Chowienczyk, R. P. Kelly, H. MacCallum et al., "Photoplethysmographic assessment of pulse wave reflection: blunted response to endothelium-dependent beta2adrenergic vasodilation in type II diabetes mellitus," Journal of the American College of Cardiology, vol. 34, no. 7, pp. 20072014, 1999.

[7] I. B. Wilkinson, I. R. Hall, H. MacCallum et al., "Pulsewave analysis: clinical evaluation of a noninvasive, widely applicable method for assessing endothelial function," Arteriosclerosis, Thrombosis, and Vascular Biology, vol. 22, no. 1, pp. 147-152, 2002.

[8] C. S. Hayward, M. Kraidly, C. M. Webband, and P. Collins, "Assessment of endothelial function using peripheral waveform analysis: a clinical application," Journal of the American College of Cardiology, vol. 40, no. 3, pp. 521-528, 2002.

[9] J. Kals, P. Kampus, M. Kals, A. Pulges, R. Teesalu, and M. Zilmer, "Effects of stimulation of nitric oxide synthesis on large artery stiffness in patients with peripheral arterial disease," Atherosclerosis, vol. 185, no. 2, pp. 368-374, 2006.

[10] S. Schroeder, M. D. Enderle, R. Ossen et al., "Noninvasive determination of endothelium-mediated vasodilation as a screening test for coronary artery disease: pilot study to assess the predictive value in comparison with angina pectoris, exercise electrocardiography, and myocardial perfusion imaging," American Heart Journal, vol. 138, no. 4, pp. 731$739,1999$.

[11] T. Heitzer, T. Schlinzig, K. Krohn, T. Meinertz, and T. Münzel, "Endothelial dysfunction, oxidative stress, and risk of cardiovascular events in patients with coronary artery disease," Circulation, vol. 104, no. 22, pp. 2673-2678, 2001.
[12] T. Neunteufl, S. Heher, R. Katzenschlager et al., "Late prognostic value of flow-mediated dilation in the brachial artery of patients with chest pain," American Journal of Cardiology, vol. 86, no. 2, pp. 207-210, 2000.

[13] Y. Inaba, J. A. Chen, and S. R. Bergmann, "Prediction of future cardiovascular outcomes by flow-mediated vasodilatation of brachial artery: a meta-analysis," International Journal of Cardiovascular Imaging, vol. 26, no. 6, pp. 631-640, 2010.

[14] M. L. Hijmering, E. S. G. Stroes, G. Pasterkamp, M. Sierevogel, J. D. Banga, and T. J. Rabelink, "Variability of flow mediated dilation: consequences for clinical application," Atherosclerosis, vol. 157, no. 2, pp. 369-373, 2001.

[15] R. J. Woodman, D. A. Playford, G. F. Watts et al., "Improved analysis of brachial artery ultrasound using a novel edgedetection software system," Journal of Applied Physiology, vol. 91, no. 2, pp. 929-937, 2001.

[16] N. M. De Roos, M. L. Bots, E. G. Schouten, and M. B. Katan, "Within-subject variability of flow-mediated vasodilation of the brachial artery in healthy men and women: implications for experimental studies," Ultrasound in Medicine and Biology, vol. 29, no. 3, pp. 401-406, 2003.

[17] H. A. Walker, G. Jackson, J. M. Ritter, and P. J. Chowienczyk, "Assessment of forearm vasodilator responses to acetylcholine and albuterol by strain gauge plethysmography: reproducibility and influence of strain gauge placement," British Journal of Clinical Pharmacology, vol. 51, no. 3, pp. 225-229, 2001.

[18] D. H. J. Thijssen, M. W. P. Bleeker, P. Smits, and M. T. E. Hopman, "Reproducibility of blood flow and postocclusive reactive hyperaemia as measured by venous occlusion plethysmography," Clinical Science, vol. 108, no. 2, pp. 151-157, 2005.

[19] M. A. Alomari, A. Solomito, R. Reyes, S. M. Khalil, R. H. Wood, and M. A. Welsch, "Measurements of vascular function using strain-gauge plethysmography: technical considerations, standardization, and physiological findings," American Journal of Physiology, vol. 286, no. 1, pp. H99H107, 2004.

[20] I. B. Wilkinsonand and D. J. Webb, "Venous occlusion plethysmography in cardiovascular research: methodology and clinical applications," British Journal of Clinical Pharmacology, vol. 52, no. 6, pp. 631-646, 2001.

[21] I. J. Kullo and A. R. Malik, "Arterial ultrasonography and tonometry as adjuncts to cardiovascular risk stratification," Journal of the American College of Cardiology, vol. 49, no. 13, pp. 1413-1426, 2007.

[22] A. J. Nelson, S. G. Worthley, J. D. Cameron et al., "Cardiovascular magnetic resonance-derived aortic distensibility: validation and observed regional differences in the elderly," Journal of Hypertension, vol. 27, no. 3, pp. 535-542, 2009.

[23] J. Ohayon, A. M. Gharib, A. Garcia et al., "Is arterial wall-strain stiffening an additional process responsible for atherosclerosis in coronary bifurcations?: an in vivo study based on dynamic CT and MRI," American Journal of Physiology, vol. 301, no. 3, pp. H1097-H1106, 2011.

[24] J. Calabia, P. Torguet, M. Garcia et al., "Doppler ultrasound in the measurement of pulse wave velocity: agreement with the Complior method," Cardiovascular Ultrasound, vol. 9, no. 1, article 13, 2011.

[25] H. B. Grotenhuis, J. J. M. Westenberg, P. Steendijk et al., "Validation and reproducibility of aortic pulse wave velocity as assessed with velocity-encoded MRI," Journal of Magnetic Resonance Imaging, vol. 30, no. 3, pp. 521-526, 2009. 
[26] B. Jiang, B. Liu, K. L. McNeill, and P. J. Chowienczyk, "Measurement of pulse wave velocity using pulse wave Doppler ultrasound: comparison with arterial tonometry," Ultrasound in Medicine and Biology, vol. 34, no. 3, pp. 509$512,2008$.

[27] A. Benetos, L. Joly, C. Perret-Guillaume et al., "Pulse wave velocity assessment by external noninvasive devices and phase-contrast magnetic resonance imaging in the obese," Hypertension, vol. 54, no. 2, pp. 421-426, 2009.

[28] T. Koivistoinen, M. Virtanen, N. Hutri-Kahonen et al., "Arterial pulse wave velocity in relation to carotid intimamedia thickness, brachial flow-mediated dilation and carotid artery distensibility: the Cardiovascular Risk in Young Finns Study and the Health 2000 Survey," Atherosclerosis, vol. 220, no. 2, pp. 387-393, 2012.

[29] E. Laffon, R. Marthan, M. Montaudon, V. Latrabe, F. Laurent, and D. Ducassou, "Feasibility of aortic pulse pressure and pressure wave velocity MRI measurement in young adults," Journal of Magnetic Resonance Imaging, vol. 21, no. 1, pp. 53$58,2005$.

[30] T. Tillin, J. Chambers, I. Malik et al., "Measurement of pulse wave velocity: site matters," Journal of Hypertension, vol. 25, no. 2, pp. 383-389, 2007.

[31] M. U. R. Naidu, B. M. Reddy, S. Yashmaina, A. N. Patnaik, and P. U. Rani, "Validity and reproducibility of arterial pulse wave velocity measurement using new device with oscillometric technique: a pilot study," BioMedical Engineering Online, vol. 4, article 49, 2005.

[32] E. M. van Leeuwen-Segarceanu, W. F. Tromp, W. J. W. Bos, O. J. M. Vogels, J. W. Groothoff, and J. H. Van Der Lee, "Comparison of two instruments measuring carotid-femoral pulse wave velocity: vicorder versus SphygmoCor," Journal of Hypertension, vol. 28, no. 8, pp. 1687-1691, 2010.

[33] I. G. Horvath, A. Nemeth, Z. Lenkey et al., "Invasive validation of a new oscillometric device (Arteriograph) for measuring augmentation index, central blood pressure and aortic pulse wave velocity," Journal of Hypertension, vol. 28, no. 10, pp. 2068-2075, 2010.

[34] S. S. Hickson, M. Butlin, J. Broad, A. P. Avolio, I. B. Wilkinson, and C. M. McEniery, "Validity and repeatability of the Vicorder apparatus: a comparison with the SphygmoCor device," Hypertension Research, vol. 32, no. 12, pp. 10791085, 2009.

[35] P. Salvi, G. Lio, C. Labat, E. Ricci, B. Pannier, and A. Benetos, "Validation of a new non-invasive portable tonometer for determining arterial pressure wave and pulse wave velocity: the PulsePen device," Journal of Hypertension, vol. 22, no. 12, pp. 2285-2293, 2004.

[36] J. Sugawara, K. Hayashi, T. Yokoi, and H. Tanaka, "Carotidfemoral pulse wave velocity: impact of different arterial path length measurements," Artery Research, vol. 4, no. 1, pp. 2731,2010

[37] T. Weber, M. Ammer, M. Rammer et al., "Noninvasive determination of carotid-femoral pulse wave velocity depends critically on assessment of travel distance: a comparison with invasive measurement," Journal of Hypertension, vol. 27, no. 8, pp. 1624-1630, 2009.

[38] Y. Matsui, K. Kario, J. Ishikawa, K. Eguchi, S. Hoshide, and K. Shimada, "Reproducibility of arterial stiffness indices (pulse wave velocity and augmentation index) simultaneously assessed by automated pulse wave analysis and their associated risk factors in essential hypertensive patients," Hypertension Research, vol. 27, no. 11, pp. 851-857, 2004.
[39] M. Munakata, N. Ito, T. Nunokawa, and K. Yoshinaga, "Utility of automated brachial ankle pulse wave velocity measurements in hypertensive patients," American Journal of Hypertension, vol. 16, no. 8, pp. 653-657, 2003.

[40] A. B. Liu, P. C. Hsu, Z. L. Chenand, and H. T. Wu, "Measuring pulse wave velocity using ECG and photoplethysmography," Journal of Medical Systems, vol. 35, no. 5, pp. 771-777, 2011.

[41] M. C. Wang, A. B. Wu, M. F. Cheng, J. Y. Chen, C. S. Ho, and W. C. Tsai, "Association of arterial stiffness indexes, determined from digital volume pulse measurement and cardiovascular risk factors in chronic kidney disease," American Journal of Hypertension, vol. 24, no. 5, pp. 544-549, 2011.

[42] J. M. Padilla, E. J. Berjano, J. Saiz, R. Rodriguezand, and L. Facila, "Pulse wave velocity and digital volume pulse as indirect estimators of blood pressure: pilot study on healthy volunteers," Cardiovascular Engineering, vol. 9, no. 3, pp. 104-112, 2009.

[43] S. R. Alty, N. Angarita-Jaimes, S. C. Millasseau, and P. J. Chowienczyk, "Predicting arterial stiffness from the digital volume pulse waveform," IEEE Transactions on Biomedical Engineering, vol. 54, no. 12, pp. 2268-2275, 2007.

[44] N. A. Jatoi, A. Mahmud, K. Bennett, and J. Feely, "Assessment of arterial stiffness in hypertension: comparison of oscillometric (Arteriograph), piezoelectronic (Complior) and tonometric (SphygmoCor) techniques," Journal of Hypertension, vol. 27, no. 11, pp. 2186-2191, 2009.

[45] J. Baulmann, U. Schillings, S. Rickert et al., "A new oscillometric method for assessment of arterial stiffness: comparison with tonometric and piezo-electronic methods," Journal of Hypertension, vol. 26, no. 3, pp. 523-528, 2008.

[46] S. A. Hope, I. T. Meredith, and J. D. Cameron, "Arterial transfer functions and the reconstruction of central aortic waveforms: myths, controversies and misconceptions," Journal of Hypertension, vol. 26, no. 1, pp. 4-7, 2008.

[47] S. Wassertheurer, J. Kropf, T. Weber et al., "A new oscillometric method for pulse wave analysis: comparison with a common tonometric method," Journal of Human Hypertension, vol. 24, no. 8, pp. 498-504, 2010.

[48] S. C. Millasseau, R. P. Kelly, J. M. Ritter, and P. J. Chowienczyk, "Determination of age-related increases in large artery stiffness by digital pulse contour analysis," Clinical Science, vol. 103, no. 4, pp. 371-377, 2002.

[49] D. Kracht, R. Shroff, and S. Baig, "Validating a new oscillometric device for aortic pulse wave velocity measurements in children and adolescents," American Journal of Hypertension, vol. 24, no. 12, pp. 1294-1299, 2011.

[50] C. Palombo, M. Kozakova, C. Morizzo et al., "Circulating endothelial progenitor cells and large artery structure and function in young subjects with uncomplicated type 1 diabetes," Cardiovascular Diabetology, vol. 10, article 88, 2011.

[51] P. Salvi, M. E. Safar, C. Labat, C. Borghi, P. Lacolley, and A. Benetos, "Heart disease and changes in pulse wave velocity and pulse pressure amplification in the elderly over 80 years: the PARTAGE Study," Journal of Hypertension, vol. 28, no. 10, pp. 2127-2133, 2010.

[52] T. Weber, S. Wassertheurer, M. Rammer et al., "Validation of a brachial cuff-based method for estimating central systolic blood pressure," Hypertension, vol. 58, no. 5, pp. 825-832, 2011.

[53] B. Gaszner, Z. Lenkey, M. Illyes et al., "Comparison of aortic and carotid arterial stiffness parameters in patients with 
verified coronary artery disease," Clinical Cardiology, vol. 35, no. 1, pp. 26-31, 2012.

[54] H. Gavaller, R. Sepp, M. Csanady, T. Forsterand, and A. Nemes, "Hypertrophic cardiomyopathy is associated with abnormal echocardiographic aortic elastic properties and arteriograph-derived pulse-wave velocity," Echocardiography, vol. 28, no. 8, pp. 848-852, 2011.

[55] M. R. Rezai, G. Goudot, C. Winters, J. D. Finn, F. C. Wuand, and J. K. Cruickshank, "Calibration mode influences central blood pressure differences between SphygmoCor and two newer devices, the Arteriograph and Omron HEM-9000," Hypertension Research, vol. 34, no. 9, pp. 1046-1051, 2011.

[56] A. Nemes, R. Takacs, H. Gavaller et al., "Correlations between aortic stiffness and parasympathetic autonomic function in healthy volunteers," Canadian Journal of Physiology and Pharmacology, vol. 88, no. 12, pp. 1166-1171, 2010.

[57] A. Nemes, R. Takacs, and H. Gavaller, "Correlations between Arteriograph-derived pulse wave velocity and aortic elastic properties by echocardiography," Clinical Physiology and Functional Imaging, vol. 31, no. 1, pp. 61-65, 2011.

[58] F. Seibert, C. Behrendt, S. Schmidt, M. van der Giet, W. Zidek, and T. H. Westhoff, "Differential effects of cyclosporine and tacrolimus on arterial function," Transplant International, vol. 24, no. 7, pp. 708-715, 2011.

[59] R. E. Climie, M. G. Schultz, S. B. Nikolic, K. D. Ahuja, J. W. Felland, and J. E. Sharman, "Validity and reliability of central blood pressure estimated by upper arm oscillometric cuff pressure," American Journal of Hypertension, vol. 25, no. 4, pp. 414-420, 2012.

[60] A. Lowe, W. Harrison, E. El-Aklouk, P. Ruygrok, and A. M. Al-Jumaily, "Non-invasive model-based estimation of aortic pulse pressure using suprasystolic brachial pressure waveforms," Journal of Biomechanics, vol. 42, no. 13, pp. 2111-2115, 2009.

[61] S. C. Millasseau, F. G. Guigui, R. P. Kelly et al., "Noninvasive assessment of the digital volume pulse: comparison with the peripheral pressure pulse," Hypertension, vol. 36, no. 6, pp. 952-956, 2000.

[62] M. F. O'Rourke and A. P. X. J. Jiang, "Pulse wave analysis," British Journal of Clinical Pharmacology, vol. 51, no. 6, pp. 507-522, 2001.

[63] W. W. Nicholsand and M. F. O'Rourke, "Ascending aortic pressure waves," in McDonald's Blood Flow in Arteries Theoretical, Experimental and Clinical Principles, W. W. Nichols and M. F. O'Rourke, Eds., Edward Arnold, London, UK, 1998.

[64] M. Karamanoglu, M. F. O’Rourke, A. P. Avolio, and R. P. Kelly, "An analysis of the relationship between central aortic and peripheral upper limb pressure waves in man," European Heart Journal, vol. 14, no. 2, pp. 160-167, 1993.

[65] C. H. Chen, E. Nevo, B. Fetics et al., "Estimation of Central aortic pressure waveform by mathematical transformation of radial tonometry pressure: validation of generalized transfer function," Circulation, vol. 95, no. 7, pp. 1827-1836, 1997.

[66] K. Takazawa, M. F. O’Rourke, M. Fujita et al., "Estimation of ascending aortic pressure from radial arterial pressure using a generalised transfer function," Zeitschrift fur Kardiologie, vol. 85, no. 3, pp. 137-139, 1996.

[67] B. Fetics, E. Nevo, C. H. Chen, and D. A. Kass, "Parametric model derivation of transfer function for noninvasive estimation of aortic pressure by radial tonometry," IEEE Transactions on Biomedical Engineering, vol. 46, no. 6, pp. 698-706, 1999.
[68] S. J. Marchais, A. P. Guerin, B. M. Pannier, B. I. Levy, M. E. Safar, and G. M. London, "Wave reflections and cardiac hypertrophy in chronic uremia: influence of body size," Hypertension, vol. 22, no. 6, pp. 876-883, 1993.

[69] J. J. Oliverand and D. J. Webb, "Noninvasive assessment of arterial stiffness and risk of atherosclerotic events," Arteriosclerosis, Thrombosis, and Vascular Biology, vol. 23, no. 4, pp. 554-566, 2003.

[70] R. P. Kelly, S. C. Millasseau, J. M. Ritter, and P. J. Chowienczyk, "Vasoactive drugs influence aortic augmentation index independently of pulse-wave velocity in healthy men," Hypertension, vol. 37, no. 6, pp. 1429-1433, 2001.

[71] I. B. Wilkinson, H. MacCallum, P. C. Hupperetz, C. J. van Thoor, J. R. Cockcroft, and D. J. Webb, "Changes in the derived central pressure waveform and pulse pressure in response to angiotensin II and noradrenaline in man," Journal of Physiology, vol. 530, no. 3, pp. 541-550, 2001.

[72] H. Smulyan, S. J. Marchais, B. Pannier, A. P. Guerin, M. E. Safar, and G. M. London, "Influence of body height on pulsatile arterial hemodynamic data," Journal of the American College of Cardiology, vol. 31, no. 5, pp. 1103-1109, 1998.

[73] I. B. Wilkinson, H. MacCallum, L. Flint, J. R. Cockcroft, D. E. Newby, and D. J. Webb, "The influence of heart rate on augmentation index and central arterial pressure in humans," Journal of Physiology, vol. 525, no. 1, pp. 263-270, 2000.

[74] C. D. Gatzka, J. D. Cameron, A. M. Dart et al., "Correction of carotid augmentation index for heart rate in elderly essential hypertensives. ANBP2 Investigators. Australian Comparative Outcome Trial of Angiotensin-Converting Enzyme Inhibitor- and Diuretic-Based Treatment of Hypertension in the Elderly," American Journal of Hypertension, vol. 14, no. 6, part 1, pp. 573-577, 2001.

[75] G. M. London, J. Blacher, B. Pannier, A. P. Guérin, S. J. Marchais, and M. E. Safar, "Arterial wave reflections and survival in end-stage renal failure," Hypertension, vol. 38, no. 3, pp. 434-438, 2001.

[76] S. Riggio, G. Mandraffino, M. A. Sardo et al., "Pulse wave velocity and augmentation index, but not intimamedia thickness, are early indicators of vascular damage in hypercholesterolemic children," European Journal of Clinical Investigation, vol. 40, no. 3, pp. 250-257, 2010.

[77] C. Russo, Z. Jin, Y. Takei et al., "Arterial wave reflection and subclinical left ventricular systolic dysfunction," Journal of Hypertension, vol. 29, no. 3, pp. 574-582, 2011.

[78] J. Y. Chen, C. H. Chou, Y. L. Lee et al., "Association of central aortic pressures indexes with development of diabetes mellitus in essential hypertension," American Journal of Hypertension, vol. 23, no. 10, pp. 1069-1073, 2010.

[79] T. Weber, M. F. O’Rourke, E. Lassnig et al., "Pulse waveform characteristics predict cardiovascular events and mortality in patients undergoing coronary angiography," Journal of Hypertension, vol. 28, no. 4, pp. 797-805, 2010.

[80] K. L. Wang, H. M. Cheng, S. H. Sung et al., "Wave reflection and arterial stiffness in the prediction of 15-year all-cause and cardiovascular mortalities: a community-based study," Hypertension, vol. 55, no. 3, pp. 799-805, 2010.

[81] T. G. Papaioannou, K. S. Stamatelopoulos, G. Georgiopoulos et al., "Arterial wave reflections during the menstrual cycle of healthy women: a reproducibility study," Hypertension, vol. 54, no. 5, pp. 1021-1027, 2009.

[82] B. Paul, C. L. Hewitson, R. J. Woodman, and A. A. Mangoni, "Analysis of short-term reproducibility of arterial vasoreactivity by pulse-wave analysis after pharmacological 
challenge," Clinical and Experimental Pharmacology and Physiology, vol. 36, no. 1, pp. 49-54, 2009.

[83] I. B. Wilkinson, S. A. Fuchs, I. M. Jansen et al., "Reproducibility of pulse wave velocity and augmentation index measured by pulse wave analysis," Journal of Hypertension, vol. 16, no. 12, part 2, pp. 2079-2084, 1998.

[84] Y. L. Liang, H. Teede, D. Kotsopoulos et al., "Non-invasive measurements of arterial structure and function: repeatability, interrelationships and trial sample size," Clinical Science, vol. 95, no. 6, pp. 669-679, 1998.

[85] A. Siebenhofer, C. R. W. Kemp, A. J. Sutton, and B. Williams, "The reproducibility of central aortic blood pressure measurements in healthy subjects using applanation tonometry and sphygmocardiography," Journal of Human Hypertension, vol. 13, no. 9, pp. 625-629, 1999.

[86] J. Filipovsky, V. Svobodovaand, and L. Pecen, "Reproducibility of radial pulse wave analysis in healthy subjects," Journal of Hypertension, vol. 18, no. 8, pp. 1033-1040, 2000.

[87] M. T. Savage, C. J. Ferro, S. J. Pinder, and C. R. V. Tomson, "Reproducibility of derived central arterial waveforms in patients with chronic renal failure," Clinical Science, vol. 103, no. 1, pp. 59-65, 2002.

[88] T. G. Papaioannou, K. S. Stamatelopoulos, E. Gialafos et al., "Monitoring of arterial stiffness indices by applanation tonometry and pulse wave analysis: reproducibility at low blood pressures," Journal of Clinical Monitoring and Computing, vol. 18, no. 2, pp. 137-144, 2004.

[89] T. Weber, J. Auer, M. F. O’Rourke et al., "Arterial stiffness, wave reflections, and the risk of coronary artery disease," Circulation, vol. 109, no. 2, pp. 184-189, 2004.

[90] E. ter Avest, S. Holewijn, A. F. H. Stalenhoef, and J. De Graaf, "Variation in non-invasive measurements of vascular function in healthy volunteers during daytime," Clinical Science, vol. 108, no. 5, pp. 425-431, 2005.

[91] T. G. Papaioannou, E. N. Karatzis, K. N. Karatzi et al., "Hourto-hour and week-to-week variability and reproducibility of wave reflection indices derived by aortic pulse wave analysis: implications for studies with repeated measurements," Journal of Hypertension, vol. 25, no. 8, pp. 1678-1686, 2007.

[92] M. Crilly, C. Coch, H. Clark, M. Bruceand, and D. Williams, "Repeatability of the measurement of augmentation index in the clinical assessment of arterial stiffness using radial applanation tonometry," Scandinavian Journal of Clinical \& Laboratory, vol. 67, no. 4, pp. 413-422, 2007.

[93] M. Crilly, C. Coch, M. Bruce, H. Clark, and D. Williams, "Indices of cardiovascular dunction derived from peripheral pulse wave analysis using radial applanation tonometry: a measurement repeatability study," Vascular Medicine, vol. 12, no. 3, pp. 189-197, 2007.

[94] M. Frimodt-Møller, A. H. Nielsen, A. L. Kamper, and S. Strandgaard, "Reproducibility of pulse-wave analysis and pulse-wave velocity determination in chronic kidney disease," Nephrology Dialysis Transplantation, vol. 23, no. 2, pp. 594-600, 2008.

[95] M. F. O'Rourke and R. P. Kelly, "Wave reflection in the systemic circulation and its implications in ventricular function," Journal of Hypertension, vol. 11, no. 4, pp. 327-337, 1993.

[96] R. Ross, "Atherosclerosis—an inflammatory disease," The New England Journal of Medicine, vol. 340, no. 2, pp. 115126, 1999.

[97] Y. Gao, "The multiple actions of NO," Pflugers Archiv European Journal of Physiology, vol. 459, no. 6, pp. 829-839, 2010 .
[98] T. Michel and P. M. Vanhoutte, "Cellular signaling and NO production," Pflugers Archiv European Journal of Physiology, vol. 459, no. 6, pp. 807-816, 2010.

[99] S. Moncadaand and E. A. Higgs, "Nitric oxide and the vascular endothelium," Handbook of Experimental Pharmacology, vol. 176, part 1, pp. 213-254, 2006.

[100] C. Napoli, F. de Nigris, S. Williams-Ignarro, O. Pignalosa, V. Sica, and L. J. Ignarro, "Nitric oxide and atherosclerosis: an update," Nitric Oxide, vol. 15, no. 4, pp. 265-279, 2006.

[101] A. Koller, D. Sun, and G. Kaley, "Role of shear stress and endothelial prostaglandins in flow- and viscosity-induced dilation of arterioles in vitro," Circulation Research, vol. 72, no. 6, pp. 1276-1284, 1993.

[102] R. M. Bryan Jr., J. You, E. M. Golding, and S. P. Marrelli, "Endothelium-derived hyperpolarizing factor: a cousin to nitric oxide and prostacyclin," Anesthesiology, vol. 102, no. 6, pp. 1261-1277, 2005.

[103] S. G. Taylor and A. H. Weston, "Endothelium-derived hyperpolarizing factor: a new endogenous inhibitor from the vascular endothelium," Trends in Pharmacological Sciences, vol. 9, no. 8, pp. 272-274, 1988.

[104] R. Joannides, J. Bellienand, and C. Thuillez, "Clinical methods for the evaluation of endothelial function-a focus on resistance arteries," Fundamental and Clinical Pharmacology, vol. 20, no. 3, pp. 311-320, 2006.

[105] J. Lekakis, P. Abraham, A. Balbarini et al., "Methods for evaluating endothelial function: a position statement from the European Society of Cardiology Working Group on Peripheral Circulation," European Journal of Cardiovascular Prevention and Rehabilitation, vol. 18, no. 6, pp. 775-789, 2011.

[106] D. S. Celermajer, K. E. Sorensen, V. M. Gooch et al., "Noninvasive detection of endothelial dysfunction in children and adults at risk of atherosclerosis," The Lancet, vol. 340, no. 8828, pp. 1111-1115, 1992.

[107] L. Stonerand and M. J. Sabatier, "Assessments of endothelial function using ultrasound," in Ultrasound, P. Ainslie, Ed., InTech, Rijeka, Croatia, 2012.

[108] I. B. Wilkinson, A. Qasem, C. M. McEniery, D. J. Webb, A. P. Avolio, and J. R. Cockcroft, "Nitric oxide regulates local arterial distensibility in vivo," Circulation, vol. 105, no. 2, pp. 213-217, 2002.

[109] J. L. Fleiss, The Design and Analysis of Clinical Experiments, John Wiley \& Sons, New York, NY, USA, 1986.

[110] M. L. Stoner, M. Erickson, J. M. Young, M. Fryerand, and M. J. Sabatier, "There's more to flow-mediated dilation than nitric oxide," Journal of Atherosclerosis and Thrombosis. In press.

[111] P. G. McLean, D. Aston, D. Sarkar, and A. Ahluwalia, "Protease-activated receptor-2 activation causes EDHFlike coronary vasodilation: selective preservation in ischemia/reperfusion injury: involvement of lipoxygenase products, VR1 receptors, and C-fibers," Circulation Research, vol. 90, no. 4, pp. 465-472, 2002.

[112] E. M. Golding, J. You, C. S. Robertson, and R. M. Bryan Jr., "Potentiated endothelium-derived hyperpolarizing factormediated dilations in cerebral arteries following mild head injury," Journal of Neurotrauma, vol. 18, no. 7, pp. 691-697, 2001.

[113] M. Malmsjo, A. Bergdahl, X. H. Zhao et al., "Enhanced acetylcholine and P2Y-receptor stimulated vascular EDHFdilatation in congestive heart failure," Cardiovascular Research, vol. 43, no. 1, pp. 200-209, 1999. 
[114] R. P. Brandes, A. Behra, C. Lebherz et al., "N(G)-nitroL-arginine- and indomethacin-resistant endothelium-dependent relaxation in the rabbit renal artery: effect of hypercholesterolemia," Atherosclerosis, vol. 135, no. 1, pp. 49$55,1997$.

[115] C. Thollon, M. P. Fournet-Bourguignon, D. Saboureau et al., "Consequences of reduced production of NO on vascular reactivity of porcine coronary arteries after angioplasty: importance of EDHF," British Journal of Pharmacology, vol. 136, no. 8, pp. 1153-1161, 2002.

[116] H. Miura, R. E. Wachtel, Y. Liu et al., "Flow-induced dilation of human coronary arterioles important role of $\mathrm{Ca} 2+-$ activated K+ channels," Circulation, vol. 103, no. 15, pp. 1992-1998, 2001.

[117] G. A. FitzGerald, B. Smith, A. K. Pedersen, and A. R. Brash, "Increased prostacyclin biosynthesis in patients with severe atherosclerosis and platelet activation," The New England Journal of Medicine, vol. 310, no. 17, pp. 1065-1068, 1984.

[118] L. Mayahi, L. Mason, K. Bleasdale-Barr et al., "Endothelial, sympathetic, and cardiac function in inherited (6R)-LErythro-5,6,7,8-Tetrahydro-L-Biopterin deficiency," Circulation, vol. 3, no. 6, pp. 513-522, 2010.

[119] B. Hornig, V. Maier, and H. Drexler, "Physical training improves endothelial function in patients with chronic heart failure," Circulation, vol. 93, no. 2, pp. 210-214, 1996.

[120] L. Ghiadoni, D. Versari, A. Magagna et al., "Ramipril dose-dependently increases nitric oxide availability in the radial artery of essential hypertension patients," Journal of Hypertension, vol. 25, no. 2, pp. 361-366, 2007. 


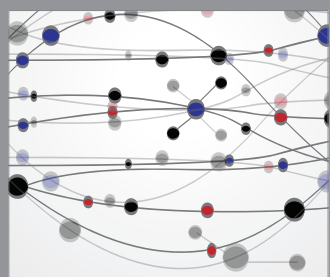

The Scientific World Journal
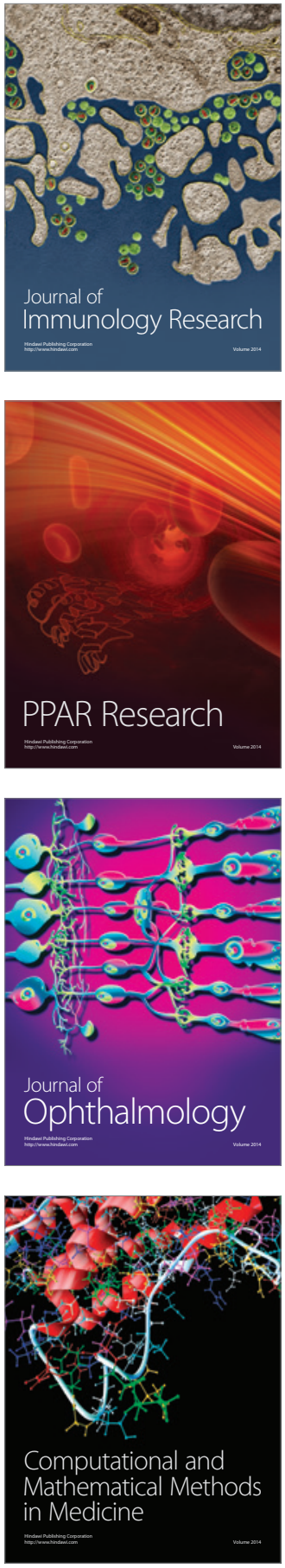

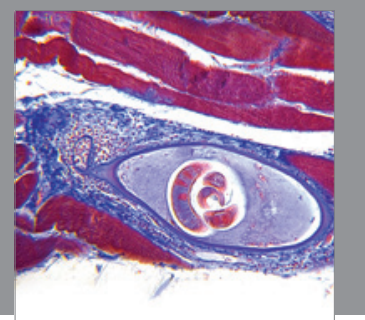

Gastroenterology

Research and Practice
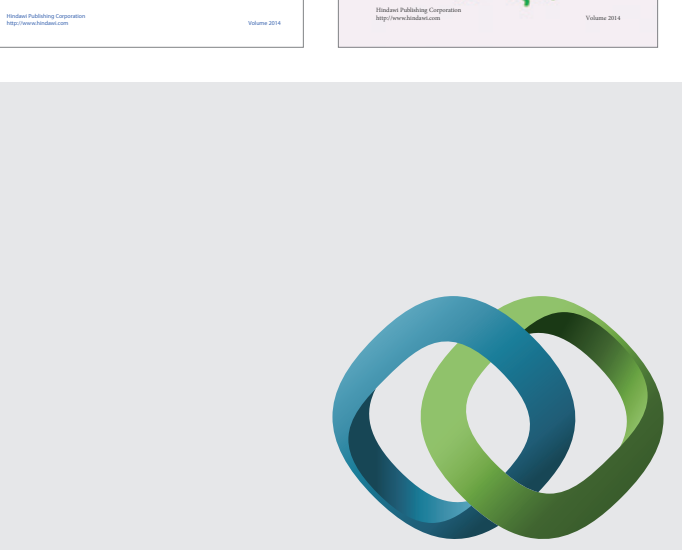

\section{Hindawi}

Submit your manuscripts at

http://www.hindawi.com
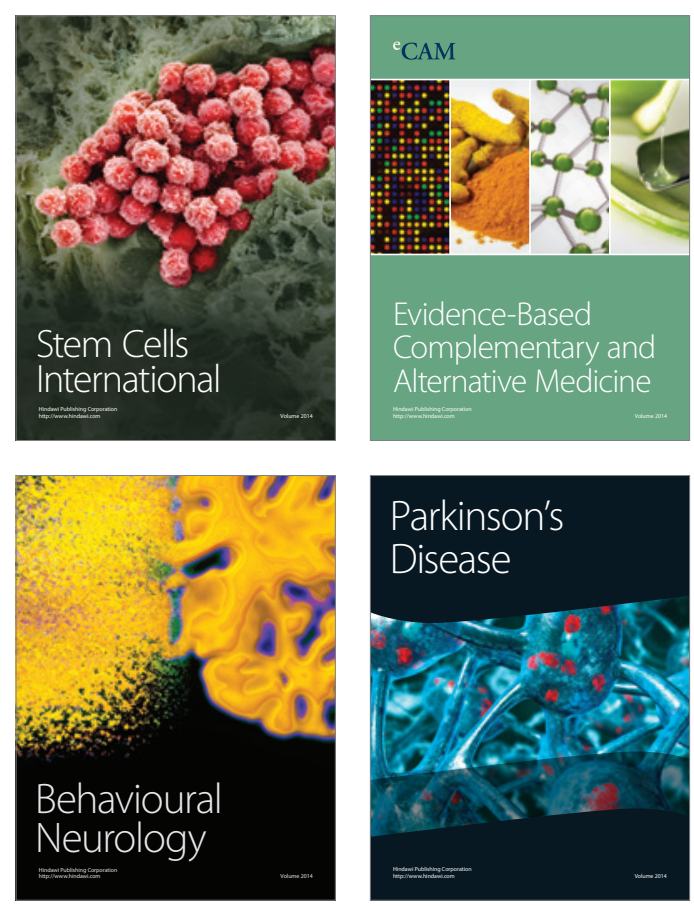

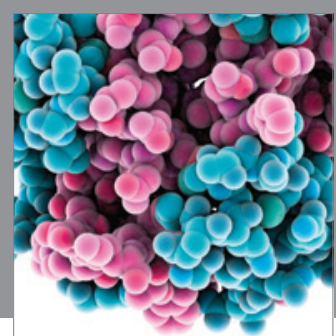

Journal of
Diabetes Research

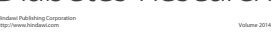

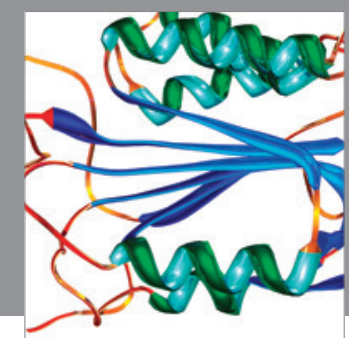

Disease Markers
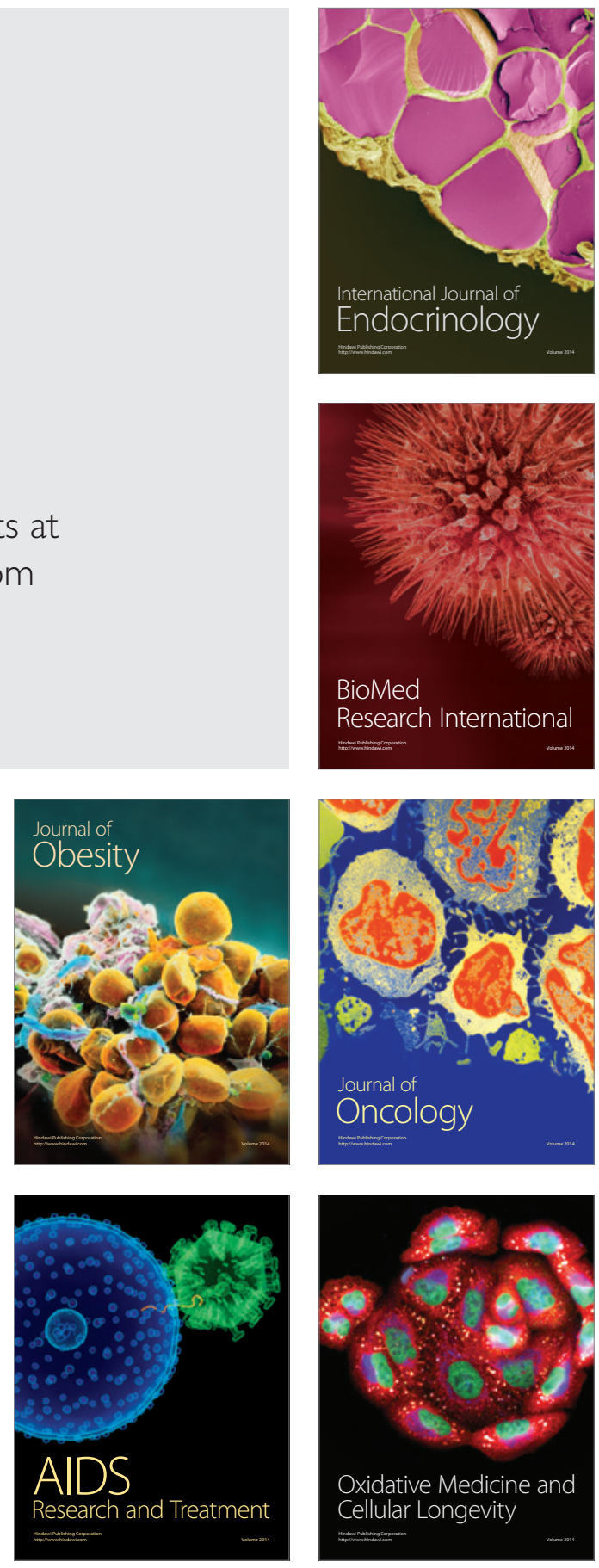